\title{
Multigrid Methods for Stabilized Nonconforming Finite El- ements for Incompressible Flow involving the Deformation Tensor Formulation
}

\author{
S. TUREK * A. OUAZZI* and R. SCHMACHTEL*
}

Received 30 April, 2002

Received in revised form 1 August, 2002

Communicated by R. Rannacher

\begin{abstract}
Edge-oriented stabilization methods in the framework of discontinuous Galerkin approaches have been recently proposed by Brenner [3] and particularly by Hansbo and Larson [5] for nonconforming finite element discretizations to satisfy a discrete Korn's inequality. We develop and analyse corresponding multigrid components in combination with local Pressure-Schur-Complement methods and give numerical examples for incompressible newtonian and non-newtonian fluids.
\end{abstract}

Keywords: Multigrid, nonconforming FEM, discontinuous Galerkin, incompressible CFD

\section{INTRODUCTION}

Nonconforming finite element methods are quite popular in the numerical treatment of incompressible flow problems, in particular due to their excellent stability properties w.r.t. the 'inf-sup-condition' and anisotropic mesh deformations. Moreover, together with discrete projection techniques or Pressure-Schur-Complement methods they provide very efficient FEM solvers for nonstationary problems [15]. And, finally, their edge-oriented degrees of freedom lead to very compact data structures which have special advantages for parallel high-performance computations [4]. Altogether, they are quite natural candidates to combine modern error control mechanisms and concepts for adaptivity in space and time with optimized high performance computing techniques to simulate realistic CFD problems.

However, for formulations including the deformation tensor $D(u)=\frac{1}{2}(\nabla u+$ $\left.(\nabla u)^{T}\right)$ which are typical for certain physical models, as for instance quasi-Newtonian flow [8], free boundaries, elasticity, etc., these approaches are unstable due to their failure to satisfy a discrete Korn's inequality [10],[11]. Based on the stabilization techniques which have been recently proposed by Brenner [3] and particularly by Hansbo and Larson [5] in a discontinuous Galerkin context by Nitsche's method, we present a numerical analysis of the resulting stability and accuracy behaviour.

\footnotetext{
* Institute of Applied Mathematics, University of Dortmund, Vogelpothsweg 87, D-44227 Dortmund, Germany

This work was supported by DFG grant No. TU102/4-1.
} 
Particularly, we explain how efficient multigrid solvers can be constructed in 2D and give examples for the numerical efficiency for realistic flow configurations. Extensions to 3D seem to be straightforward.

\subsection{Physical Models}

A fluid is called quasi-Newtonian if the Cauchy stress tensor is given by

$$
\sigma=2 v\left(D_{I I}(u)\right) D(u)-p I,
$$

where $p$ is the pressure; $D(u)=\frac{1}{2}\left(\nabla u+(\nabla u)^{T}\right)$ is the deformation rate tensor, $u$ being the velocity; $v(\cdot)$ is the nonlinear viscosity which may depend on the second invariant of the deformation rate tensor

$$
D_{I I}(u)=\frac{1}{2} D(u): D(u)=\frac{1}{2} \sum_{i, j} D_{i, j}(u) D_{i, j}(u) .
$$

Depending on the specific viscosity function $v(z)$ (with $z=D_{I I}(u)$ ) we obtain:

- Power law models of type $v(z)=v_{0} z^{\frac{r}{2}-1} \quad\left(v_{0}, r \in \mathbb{R}\right)$

- Carreau law models with $v(z)=v_{\infty}+\left(v_{0}-v_{\infty}\right)(1+\lambda z)^{\frac{r}{2}-1}\left(v_{0}, v_{\infty}, \lambda, r \in \mathbb{R}\right)$

- Schaeffer model [13] (with pressure $p$ ) for granular flow with $v(z)=p z^{-\frac{1}{2}}$

Altogether, the velocity $u$ and the pressure $p$ satisfy the following generalized incompressible Navier-Stokes equations:

$$
u_{t}+u \cdot \nabla u-\nabla \cdot\left[2 v\left(D_{I I}(u)\right) D(u)\right]+\nabla p=f \quad, \quad \nabla \cdot u=0
$$

In this paper, we will concentrate on the case of steady configurations, that means we omit the term $u_{t}$ in (1.3), and the typical Re numbers will be of moderate size. Further extensions of these nonlinear models may also have the form $v=v(D(u), p, T)$, where the computation of the temperature $T$ is integrated via a Boussinesq model.

\section{STABILIZED NONCONFORMING FINITE ELEMENTS}

\subsection{Quadrilateral Rotated Multilinear Elements}

We consider a subdivision $\tau_{h}$, consisting of quadrilaterals $\tau$, of a bounded region $\Omega \subset \mathbb{R}^{2}$ and employ the mixed Rannacher-Turek element [12],[15]. For any element $\tau$, let $(\xi, \eta)$ denote a coordinate system obtained by joining the midpoints of the opposing faces of $\tau$. On each element $\tau$ we set:

$$
\tilde{Q}_{1}:=\operatorname{span}\left\{1, \xi, \eta, \xi^{2}-\eta^{2}\right\}
$$


The degrees of freedom for the velocity are determined by the nodal functionals $\left\{F_{\Gamma}^{(a, b)}(\cdot), \Gamma \subset \partial \tau_{h}\right\}$, with

$$
F_{\Gamma}^{a}:=|\Gamma|^{-1} \int_{\Gamma} v d \gamma \quad \text { or } \quad F_{\Gamma}^{b}:=v\left(m_{\Gamma}\right) \quad\left(m_{\Gamma} \text { midpoint of edge } \Gamma\right),
$$

such that the discrete velocities belong to the following FEM space $W_{h}^{a, b}$ :

$$
W_{h}^{a, b}:=\left\{\begin{array}{l}
v_{h} \in L_{2}(\Omega) \mid v_{h \mid \tau} \in \tilde{Q}_{1}(\tau), \forall \tau \in \tau_{h}, v_{h} \text { is continuous w.r.t. } \\
\text { all nodal functionals } F_{\Gamma_{i, j}^{a, b}}^{a,}(\cdot), \forall \text { interior edges } \Gamma_{i, j}, \\
\text { and } F_{\Gamma_{i 0}}^{a, b}\left(v_{h}\right)=0, \forall \text { boundary edges } \Gamma_{i 0}
\end{array}\right\}
$$

The corresponding pressure will be approximated in the space

$$
L_{h}:=\left\{q_{h} \in L^{2}(\Omega) \mid q_{h \mid \tau}=\text { const. }, \forall \tau \in \tau_{h}\right\}
$$

While it is well-known that this finite element satisfies the BB condition, even on anisotropic meshes including large mesh deformations (see [2],[12],[15]), its approximation properties for the given cases including the deformation tensor are not clear. If we apply an appropriate linearization technique of Newton-type, then the standard FEM approach for the resulting linear problems leads to a discrete system of equations which can be written in matrix-vector form:

$$
A\left(u^{l}\right) u+B p=f \quad, \quad B^{T} u=0
$$

Here, $A\left(u^{l}\right)$ and $B$ are defined as follows for any given state $u^{l} \in W_{h}^{a, b}$ :

$$
\begin{gathered}
\left\langle A\left(u^{l}\right) u, v\right\rangle=\sum_{\tau \in \tau_{h}} \int_{\tau} 2 v\left(D_{I I}\left(u^{l}\right)\right) D(u): D(v) d x \quad \forall u, v \in W_{h}^{a, b} \\
\langle B q, v\rangle=\sum_{\tau \in \tau_{h}} \int_{\tau} q \nabla \cdot v d x \quad \forall q \in L_{h}, v \in W_{h}^{a, b}
\end{gathered}
$$

\subsection{Korn's Inequality}

Theorem 2.1. (Korn's Inequality) Let $\Omega$ be a domain in $\mathbb{R}^{2}$. Then, for all $v \in$ $H^{1}(\Omega)^{2}$, there exists a constant $c>0$ such that

$$
\|v\|_{H^{1}(\Omega)} \leqslant c\left(\|v\|_{0, \Omega}^{2}+\|D(v)\|_{0, \Omega}^{2}\right)^{\frac{1}{2}} .
$$

Definition 2.1. A discrete analogue to Korn's inequality (2.8) reads:

$$
\sum_{\tau \in \tau_{h}}\left\|v_{h}\right\|_{H^{1}(\tau)} \leqslant c\left(\sum_{\tau \in \tau_{h}}\left\|v_{h}\right\|_{0, \tau}^{2}+\left\|D\left(v_{h}\right)\right\|_{0, \tau}^{2}\right)^{\frac{1}{2}} \quad \forall v_{h} \in W_{h}^{a, b} .
$$


The continuity requirement across edges of the triangulation for the RannacherTurek element is weakened to the validity of a patch test of order one only, so that the discrete Korn's inequality (2.9) fails [10]. Another perspective is to point out the argument given by Hansbo [5] referring to Hughes [9] for the Crouzeix-Raviart element: prescribing the displacement along one side of an element means prescribing the displacement in only one node, which cannot preclude rigid body rotation. To avoid this problem, we follow the ideas of Brenner [3] and Hansbo/Larson [5],[6] to use the nonconforming finite elements in the framework of discontinuous Galerkin approximations by Nitsche's method. Hence, we add the following bilinear form for stabilization,

$$
\sum_{E \in E_{I} \cup E_{D}} \frac{1}{|E|} \int_{E}\left[\varphi_{i}\right]\left[\varphi_{j}\right] d \sigma,
$$

for all basis functions $\varphi_{i}$ and $\varphi_{j}$ of $W_{h}^{a, b}$, with can be weighted by an additional relaxation parameter $s=s(v)$ which will act as 'free' stabilization parameter. Then, the corresponding matrix $S$ is defined as:

$$
\langle S u, v\rangle=s \sum_{E \in E_{I} \cup E_{D}} \frac{1}{|E|} \int_{E}[u][v] d \sigma \quad \forall u, v \in W_{h}^{a, b}
$$

The jump of a function $u$ on an edge $\mathrm{E}$ is given by

$$
[u]= \begin{cases}u^{+} \cdot n^{+}+u^{-} \cdot n^{-} & \text {on internal edges } E_{I}, \\ u \cdot n & \text { on Dirichlet boundary edges } E_{D}, \\ 0 & \text { on Neumann boundary edges } E_{N},\end{cases}
$$

where $n$ is the outward normal to the edge and $(\cdot)^{+}$and $(\cdot)^{-}$indicate the value of the generic quantity $(\cdot)$ on the two elements sharing the same edge.

\subsection{Error Estimates and Robustness}

In the following, we present a priori error estimates for the linear (Stokes) case [5],[15], which means that we set $A:=A\left(u^{l}=0\right)$ in (2.6); the nonlinear case can be treated in the same manner following Baranger and Najib [1]. Additionally, we assume the standard quasi-uniform meshes for the analysis. Since all numerical tests indicate that analogous results are valid for anisotropic meshes, too, we expect to prove the corresponding results in near future.

First, we introduce the mesh dependent energy norm on the space $W_{h}^{a, b}$

$$
\|\mid\| \|^{2}:=\sum_{\tau \in \tau_{h}} \int_{\tau} D(v): D(v) d x+h^{-1} \sum_{E \in E_{I} \cup E_{D}} \int_{E}[v][v] d \sigma
$$

and

$$
\|q\|_{L_{h}}^{2}=\sum_{\tau \in \tau_{h}}\|q\|_{L^{2}(\tau)}^{2}
$$

on the pressure space $L_{h}$. Then, there holds [3],[5]: 


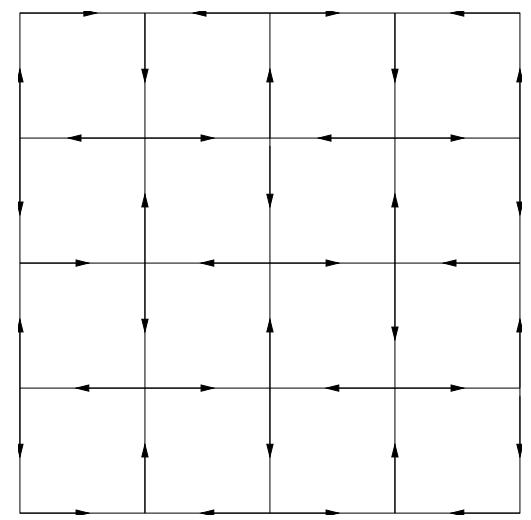

Figure 1. The specific kernel function on an equidistantly refined unit square.

Lemma 2.1. There exists a constant $c$, independent of $h$ and $v$, such that

$$
\sum_{\tau \in \tau_{h}}\|v\|_{H^{1}(\tau)}^{2} \leqslant c\|\| v \|^{2} \quad \text { for all } v \in W_{h}^{a, b}
$$

Theorem 2.2. There exist constants $\alpha, \beta$ and $C$ strictly positive and independent of $h$ such that (in the linear Stokes case) there holds $\forall v \in W_{h}^{a, b}$ :

$$
\alpha\|\| v\|\|^{2} \leqslant\langle(A+S) v, v\rangle \quad, \quad \beta \leqslant \inf _{q \in L_{h}} \sup _{v \in W_{h}^{a, b}} \frac{\langle B v, q\rangle}{\|\| v\|\|\|q\|_{L_{h}}}
$$

Moreover, assuming sufficient regularity of the solution, we have the error estimate:

$$
\left\|u-u_{h} \mid\right\|+\left\|p-p_{h}\right\|_{L_{h}} \leqslant C h\left(\|u\|_{\left.H^{2}(\Omega)\right)}+\|p\|_{H^{1}(\Omega)}\right)
$$

As a first test, we introduce the characteristic kernel function on the unit square (see [10]) which takes the values -1 or 1 in the midpoints (see Figure 1). If $u=\sum_{i} X_{i} \varphi_{i}$ (with $\varphi_{i}$ being the edge-oriented FEM basis functions in $W_{h}^{a, b}, X_{i}$ being the coefficients) we can define the (semi-)norm $\|u\|_{M}=\left(\sum_{i, j} M_{i j} X_{i} X_{j}\right)^{\frac{1}{2}}$ for any (positive semidefinite) matrix $\mathrm{M}$, and we introduce the constant $C_{M}=\frac{\|u\|_{M}}{\|u\|_{G}}$. Let $G, T$ and $S T$ be the stiffness matrices resulting from the discretization including the gradient, tensor and stabilized tensor operator respectively. Then, Table 1 clearly demonstrates that $C_{T}=0$, in contrast to $C_{S T}=O(1)$. 
Table 1.

The specific kernel function and examples for the constant in Korn's inequality on an equidistantly refined unit square.

\begin{tabular}{crccc}
\hline Level & NEL & $\|u\|_{G}$ & $\|u\|_{T}$ & $\|u\|_{S T}$ \\
\hline 2 & 4 & 0.9 & $0.63 \mathrm{E}-15$ & 0.30 \\
3 & 16 & 1.1 & $0.20 \mathrm{E}-14$ & 0.60 \\
4 & 64 & 1.3 & $0.41 \mathrm{E}-14$ & 0.76 \\
5 & 256 & 1.3 & $0.69 \mathrm{E}-14$ & 0.85 \\
6 & 1024 & 1.4 & $0.14 \mathrm{E}-14$ & 0.90 \\
7 & 4096 & 1.4 & $0.30 \mathrm{E}-13$ & 0.92 \\
8 & 16384 & 1.4 & $0.61 \mathrm{E}-13$ & 0.93 \\
\hline
\end{tabular}

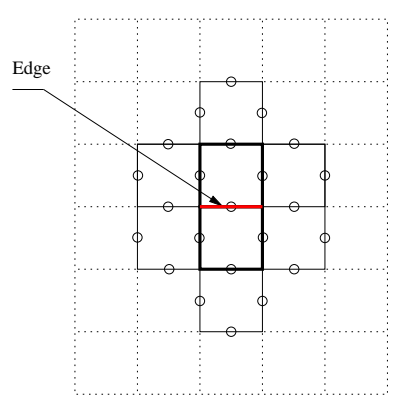

Figure 2. Involved edges for each basis function.

\section{LINEAR MULTIGRID SOLVERS}

\subsection{Sparsity of the Matrix}

Sparse matrices are an integral part of the finite element analysis for incompressible flow which may lead to huge (nonlinear) ill-conditioned systems so that very fast solvers of multigrid type are required. However, the new edge-oriented stabilization techniques destroy the typical local sparsity properties. To be more specific, the new edge-oriented stabilization involves more than the adjacent elements as can be seen in Figure 2 which shows the increased local support of basis functions in the framework of the introduced stabilization: The corresponding rows and columns for the new stiffness matrices $S$ may contain 23 non-zero matrix elements, in contrast to the usual 7 for the non-stabilized case in 2D.

To overcome the problem of storing the new matrix $S$ with regard to the standard FEM data structures, we write the matrix $S$ as a sum of two matrices $S^{*}$ and $S_{\text {rest }}$,

$$
S=S^{*}+S_{\text {rest }}
$$

where $S^{*}$ has the same sparsity structure as the usual corresponding finite element matrix; then, $S_{\text {rest }}=S-S^{*}$. Hence, $S^{*}$ can be handled with the same linear algebra 
techniques which are typically used for the treatment of the standard nonconforming finite element approach; $S_{\text {rest }}$ is the complementary part and will be used as a correction for the calculation of the residuals inside of the linear solvers only. Then, given any approximation $v$, we can write the complete residual as:

$$
f-(A+S) v=f-\left(A+S^{*}\right) v-S_{\text {rest }} v
$$

Consequently, we store in the complete stiffness matrix only the partial matrix $A+$ $S^{*}$ so that the first part of the residual can be obtained via standard matrix-vector multiplication while the second part is assembled via elementwise operations only. Moreover, the construction of preconditioners for the corresponding linear systems will only include parts of the (sub)matrix $A+S^{*}$, too, which will be explained in the following.

\subsection{The Vanka Smoother as Defect Correction Scheme}

The 'Vanka smoother' [16] as typical candidate for a local Pressure-Schur-Complement approach (see [15] for the details) is a simple iterative relaxation method for coupled systems which is acting directly on element level and which is embedded into an outer block Jacobi/Gauss-Seidel iteration. The local character of this procedure together with a global defect correction mechanism is crucial for our approach. If we denote by $\operatorname{Res}_{u}$ and $\operatorname{Res}_{p}$ the residuals for the (complete) momentum and continuity equation which include the complete stabilization term due to $S$ as described in (3.2), one basic iteration step - that is one smoothing step - can be described as:

$$
\left[\begin{array}{c}
u^{n+1} \\
p^{n+1}
\end{array}\right]=\left[\begin{array}{c}
u^{n} \\
p^{n}
\end{array}\right]+\omega^{n} \sum_{i}\left(\begin{array}{cc}
A_{\mid \Omega_{i}}+S_{\mid \Omega_{i}}^{*} & B_{\mid \Omega_{i}} \\
B_{\mid \Omega_{i}}^{T} & 0
\end{array}\right)^{-1}\left[\begin{array}{l}
\operatorname{Res}_{u}\left(u^{n}, p^{n}\right) \\
\operatorname{Res}_{p}\left(u^{n}, p^{n}\right)
\end{array}\right]
$$

In our case, the patches $\Omega_{i}$ consist of only one element which means that we restrict the global stiffness matrix to the single cells/quadrilaterals of the mesh and solve the corresponding algebraic problem. As can be seen, for the preconditioning step only parts of the matrix (here: $A+S^{*}$ ) are involved while the residual contains all parts of the matrix. Consequently, if this approach converges, the result is the solution of the stabilized version while the preconditioning steps only determine the speed of the overall iteration procedure.

Beside the explained smoother, all other multigrid components (intergrid transfer, coarse grid operators and coarse grid solvers, step-length control) are the standard ones and a detailed description can be found in [15].

\section{NUMERICAL RESULTS}

We present some results from numerical experiments with different physical models in which the computational domain is a channel geometry with an obstacle as shown in Figure 3. 


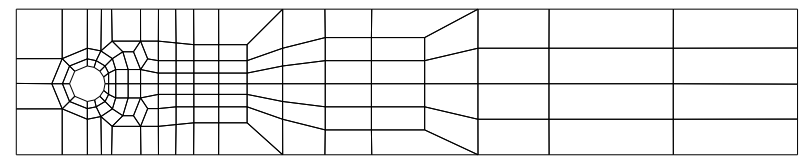

Figure 3. The coarse mesh for the 'flow around a cylinder' configuration.

Table 2.

Lift and drag coefficients for the 'flow around a cylinder' configuration.

\begin{tabular}{rcccc}
\hline $1 / v$ & & Gradient & Tensor & Stab. Tensor \\
\hline 1 & drag & $0.31252+4$ & $0.31221+4$ & $0.31231+4$ \\
& lift & $0.30898+2$ & $0.30924+2$ & $0.30936+2$ \\
\hline 10 & drag & $0.31258+3$ & $0.31227+3$ & $0.31237+3$ \\
& lift & $0.36832+1$ & $0.36851+1$ & $0.36864+1$ \\
\hline 100 & drag & $0.32016+2$ & $0.31981+2$ & $0.31990+2$ \\
& lift & $0.79786+0$ & $0.79758+0$ & $0.79770+0$ \\
\hline 1000 & drag & $0.55657+1$ & $0.55531+1$ & $0.55535+1$ \\
& lift & $0.10180-1$ & $0.10259-1$ & $0.10277-1$ \\
\hline & & & &
\end{tabular}

On the boundaries, we prescribe no-slip conditions on the walls and on the cylinder while a parabolic inflow profile is defined on the upstream boundary. At the downstream part, we prescribe natural boundary conditions (see [7]) which leads to the well-known outflow boundary condition $v D(u) \cdot n-p n=0$ (Remark: according to [7], this condition is not satisfied for parallel flow).

\subsection{Numerical Study of the Accuracy}

First of all, we want to check if the new stabilization term $S$ preserves the accuracy of the nonconforming finite element in CFD simulations. Therefore, we calculate in Table 2 the corresponding lift and drag forces acting on the cylinder for different Re numbers (here: for different viscosity parameters $v$ ) and different formulations (gradient, tensor and stabilized tensor formulation). We only show the results on level 5 (after 4 successive refinements of the given coarse mesh in Figure 3) since no major differences occur for finer or coarser meshes; we choose $s=0.5$ as damping parameter in (2.11). In addition, we show in Table 3 the corresponding results when we approximate a prescribed polynomial solution for $u$ and $p$.

Surprisingly, we do not see any significant difference between the different formulations! The discussed kernel function and hence the failure of Korn's inequality has - more or less - no visible influence on the approximation properties which is understandable in our case of (partial) Dirichlet boundary conditions: Then, no 'free' 
Table 3.

$L^{2}$ - and $H^{1}$-errors for a given polynomial solution for the 'flow around a cylinder' configuration.

\begin{tabular}{cccc}
\hline Level & Norm & Tensor & Stabilized Tensor \\
\hline \multirow{2}{*}{3} & $\left\|u-u_{h}\right\|_{1, \Omega}$ & $1.8780-1$ & $1.8903-1$ \\
& $\left\|u-u_{h}\right\|_{0, \Omega}$ & $2.1924-2$ & $2.2278-2$ \\
\hline \multirow{2}{*}{4} & $\left\|u-u_{h}\right\|_{1, \Omega}$ & $9.3418-2$ & $9.3755-2$ \\
& $\left\|u-u_{h}\right\|_{0, \Omega}$ & $5.4160-3$ & $5.4675-3$ \\
\hline \multirow{2}{*}{5} & $\left\|u-u_{h}\right\|_{1, \Omega}$ & $4.6624-2$ & $4.6711-2$ \\
& $\left\|u-u_{h}\right\|_{0, \Omega}$ & $1.3482-3$ & $1.3552-3$ \\
\hline \multirow{2}{*}{6} & $\left\|u-u_{h}\right\|_{1, \Omega}$ & $2.3298-2$ & $2.3320-2$ \\
& $\left\|u-u_{h}\right\|_{0, \Omega}$ & $3.3659-4$ & $3.3750-4$ \\
\hline
\end{tabular}

rigid body movement can occur! However, as the following calculations will show, the problem with Korn's inequality is clearly visible if we look at the corresponding behaviour of the (multigrid) solvers.

\subsection{Numerical Study of the Influence of Convection}

The following numerical experiments investigate the influence of the convective term which is treated via FEM upwinding (see [15]). For this purpose we increase the Reynolds number and list the number of resulting nonlinear iterations (NNL) and the averaged number of multigrid sweeps per nonlinear iteration (AVMG). Table 4 shows that increasing the Re number leads to 'improvements' for the multigrid solver for the (unstabilized) deformation tensor formulation (keep in mind that the case of $1 / v=1000$ corresponds to $R e=20$ only, compare with the settings for the 'DFG-Benchmark' [14]). However, in the case of very low Re numbers, the failure of Korn's inequality gets clearly visible since the averaged number of linear multigrid sweeps AVMG has to be massively increased to satisfy a desired stopping criterion.

The main result is that for Re numbers of moderate size $(R e \geqslant 10)$ the presence of the convective term seems to stabilize the multigrid behaviour since the influence of the kernel function due to the deformation tensor formulation is weakened. However, since the corresponding 'elliptic' differential operator - being of second order - is the dominating operator for small mesh width, we can see that the convergence behaviour deteriorates with finer mesh widths. This can be shown much better in the case of very small Re numbers ('Stokes' case) where the multigrid convergence behaviour is extremely mesh dependent so that the multigrid solver looses all nice features. In contrast, the introduced edge-oriented stabilization techniques together with the described multigrid smoothers and data structures lead to significant improvements so that no differences between 'stable' gradient formulation and stabilized tensor formulation are visible. 
Table 4.

Nonlinear iterations (NNL)/Averaged multigrid sweeps (AVMG) per nonlinear iteration for different Re numbers $(1 / v)$, various formulations (gradient, tensor, stabilized tensor), several levels of refinement (LEV) and varying number of smoothing steps (NSM). For very low Re numbers, neglecting the influence of the convection, the original tensor formulation exhibits severe convergence problems for the linear multigrid solvers while the gradient formulation and the proposed stabilization for the tensor formulation show the typical excellent multigrid convergence behaviour.

\begin{tabular}{cccccccc}
\hline LEV & NSM & grad & tensor & stab & grad & tensor & stab \\
\hline & \multicolumn{3}{c}{$1 / v=1$} & \multicolumn{4}{c}{$1 / v=1000$} \\
\hline 3 & 2 & $3 / 5$ & $4 / 57$ & $3 / 6$ & $10 / 7$ & $10 / 6$ & $10 / 7$ \\
& 4 & $3 / 3$ & $4 / 29$ & $3 / 3$ & $10 / 5$ & $10 / 5$ & $10 / 5$ \\
\hline 4 & 2 & $3 / 6$ & $4 / 167$ & $3 / 6$ & $11 / 6$ & $11 / 10$ & $11 / 5$ \\
& 4 & $3 / 3$ & $4 / 86$ & $3 / 3$ & $11 / 4$ & $11 / 5$ & $11 / 4$ \\
\hline 5 & 2 & $3 / 7$ & $4 / 478$ & $3 / 6$ & $12 / 5$ & $12 / 23$ & $12 / 4$ \\
& 4 & $3 / 4$ & $4 / 231$ & $3 / 4$ & $12 / 4$ & $12 / 10$ & $12 / 4$ \\
\hline 6 & 2 & $3 / 9$ & $6 / 382$ & $3 / 7$ & $12 / 4$ & $12 / 4$ & $12 / 4$ \\
& 4 & $3 / 5$ & $5 / 326$ & $3 / 4$ & $12 / 3$ & $12 / 3$ & $12 / 3$ \\
\hline
\end{tabular}

However, we also have to remark that the inclusion of the partial matrix $S^{*}$ into the preconditioning matrix $A+S^{*}$ is necessary. It might appear to be much easier for the implementation procedure to employ only the partial matrix $A$ in the preconditioning step since this approach does not require any modification of the 'Vanka smoother'; only the defect calculation has to be adapted. However, in this case, the solver behaves more like an (incomplete) block-Jacobi iteration so that convergence problems may arise as soon as significant mesh anisotropies occur. Therefore, we strongly recommend to include the partial matrix $S^{*}$ from the stabilization procedure into the preconditioning approach.

\subsection{Numerical Study of the Size of the Stabilization Parameter}

In the next simulations, we consider the case of (nonlinear) quasi-Newtonian flow where we employ the described Power law model for the viscosity function $v$. However, in this case, we are confronted with the question of how to choose the relaxation parameter $s$ in the described stabilization approach which should be related to the (local) size of the function $v=v\left(D_{I I}(u)\right)$. In the following, we prescribe $v(D)=v_{0}(\varepsilon+\|D\|)^{\alpha}$ which leads to 'shear-thickening' $(\alpha \geqslant 0)$ or 'shear-thinning' $(\alpha \leqslant 0)$. For the special parameter choices $\varepsilon=10^{-1}, v_{0}=1$ and $|\alpha|=0.7$, the viscosity function $v$ varies locally by a factor of $10(\alpha=0.7)$ or even $30(\alpha=-0.7)$. In the following, we denote this factor by F.C.V. ('Factor for Changes of Viscosity'). Figure 4 shows corresponding plots of the resulting viscosity function $v\left(D_{I I}(u)\right)$ for the converged solution $u$ of this nonlinear problem. 


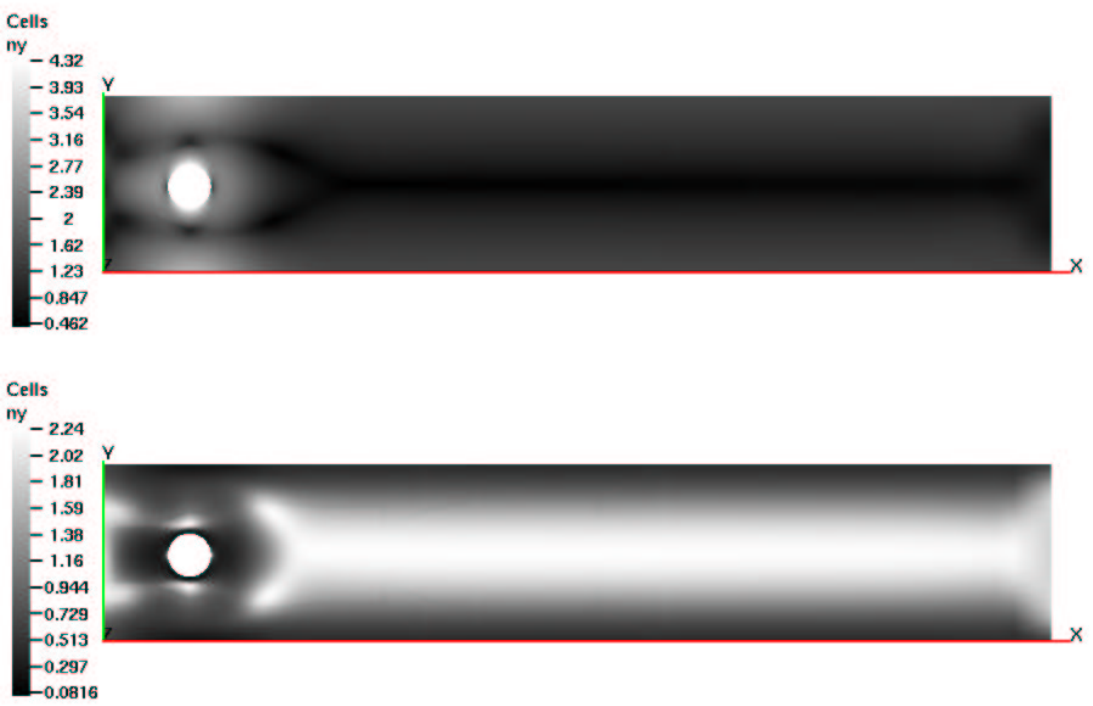

Figure 4. The resulting viscosity for the described cases of shear thickening/thinning.

Looking at this locally changing behaviour of the viscosity function, the question arises of how to choose the stabilization parameter $s=s(v)$. In the following Table 5 we show results for the version $s_{\text {const }}$ in which case $s$ was fixed to be the minimum value of $v$. In contrast, the approach $s_{\text {local }}$ is based on the local size of the viscosity $v$ on every edge and takes into account the local variations due to changes w.r.t. the value of $D_{I I}(u)$. As expected, the locally adapted choice $s_{\text {local }}$ shows a better nonlinear convergence behaviour (number of nonlinear iterations NNL), and the corresponding linear multigrid behaviour (averaged number of multigrid sweeps per nonlinear cycle AVMG) is significantly better, due to a possible underestimation of the value $s_{\text {const }}$ in the stabilization procedure; the case $s=0$ (no stabilization at all) shows the expected problems due to the failure of Korn's inequality.

Additionally, we discuss another quantitative aspect of selecting the appropriate stabilization parameter which can be represented in the form $s=\omega \cdot s_{\text {const }}$, resp., $s=\omega \cdot s_{\text {local }}$. Then, we vary the parameter $\omega$. The results in Table 5 demonstrate again the improved behaviour of the locally adaptive stabilization parameter $s_{\text {local }}$, however they also show that the right quantitative choice of the absolute size due to the additional damping parameter $\omega$ tends to be crucial so that further research is required for 'optimal' choices of the stabilization parameter $s$.

Finally, we show typical results for varying the parameters $\varepsilon$ and particularly $\alpha$ for the shear-thinning case. With $\alpha$ approaching -1 (and $\varepsilon$ tending to 0 ), the nonlinearity gets stronger and correspondingly the 'local changes of viscosity' (F.C.V.) significantly increase. Nevertheless, the local adaptive choice $s_{\text {local }}$ for the stabilization parameter has no problem at all (NNL increases due to a more dominant 
Table 5.

NNL/AVMG for different choices of stabilization $\left(\varepsilon=10^{-1}, v_{0}=1\right)$ for $v(D)=v_{0}(\varepsilon+\|D\|)^{\alpha}$.

\begin{tabular}{ccccccc}
\hline \multicolumn{3}{c}{$\alpha=-0.7$} & \multicolumn{3}{c}{$\alpha=0.7$} \\
\hline Level & $s=0$ & $s_{\text {const }}$ & $s_{\text {local }}$ & $s=0$ & $s_{\text {const }}$ & $s_{\text {local }}$ \\
\hline 3 & $26 / 48$ & $24 / 4$ & $24 / 3$ & $17 / 7$ & $17 / 6$ & $17 / 5$ \\
4 & $26 / 140$ & $25 / 5$ & $25 / 3$ & $18 / 15$ & $17 / 8$ & $17 / 5$ \\
5 & $27 / 414$ & $24 / 6$ & $24 / 3$ & $50 / 212$ & $17 / 10$ & $17 / 5$ \\
6 & - & $24 / 7$ & $24 / 3$ & - & $17 / 12$ & $17 / 5$ \\
\hline & & & Level 4 & & & \\
$\omega$ & 0.1 & 0.01 & 0.001 & 0.1 & 0.01 & 0.001 \\
\hline$\omega \cdot s_{\text {const }}$ & $25 / 4$ & $25 / 11$ & $25 / 15$ & $17 / 6$ & $17 / 14$ & $17 / 27$ \\
$\omega \cdot s_{\text {local }}$ & $25 / 3$ & $25 / 3$ & $25 / 8$ & $17 / 5$ & $17 / 10$ & $17 / 23$ \\
\hline
\end{tabular}

Table 6.

Convergence behaviour (NNL/AVMG) for growing F.C.V. factors for $v(D)=$ $v_{0}(\varepsilon+\|D\|)^{\alpha}$.

\begin{tabular}{ccccccc}
\hline & \multicolumn{2}{c}{$\begin{array}{c}\varepsilon=10^{-1} \\
\alpha=-0.6\end{array}$} & \multicolumn{2}{c}{$\begin{array}{c}\varepsilon=10^{-2} \\
\alpha=-0.7\end{array}$} & \multicolumn{2}{c}{$\begin{array}{c}\varepsilon=10^{-3} \\
\alpha=-0.8\end{array}$} \\
\hline Level & tensor & stab. tensor & tensor & stab. tensor & tensor & stab. tensor \\
\hline 3 & $18 / 7$ & $17 / 3$ & $26 / 6$ & $24 / 3$ & $41 / 21$ & $37 / 3$ \\
4 & $18 / 19$ & $18 / 3$ & $25 / 19$ & $25 / 3$ & $39 / 18$ & $37 / 4$ \\
5 & $18 / 53$ & $18 / 3$ & $24 / 51$ & $25 / 3$ & $38 / 49$ & $38 / 4$ \\
\hline F.C.V. & 14 & & 62 & & 362 \\
\hline
\end{tabular}

nonlinearity while AVMG remains constant; here, a better nonlinear Newton-like solver instead of the fixed-point-like iteration is under research) while the unstabilized tensor formulation exhibits severe convergence problems for the involved linear multigrid steps.

\section{CONCLUDING REMARKS AND OUTLOOK}

The aim of this work was to highlight the flexibility, robustness and efficiency of multigrid solvers for stabilized nonconforming finite element discretizations to simulate general incompressible flow problems involving the deformation tensor. Based on the work by Brenner [3] and Hansbo and Larson [5] who have introduced and analyzed appropriate edge-oriented stabilization techniques by discontinuous Galerkin approaches in the context of Nitsche's method, we have concentrated on the corresponding (multigrid) solver and aspects of appropriate data structures to include these discretization techniques in standard FEM codes. The results are very promising and show that the typical efficiency of multigrid solvers for the gradient formulation can be transferred to formulations including the deformation tensor. The major 
task is to modify existing local Multilevel-Pressure-Schur-Complement smoothers ('Vanka smoother') [15] in the framework of defect correction approaches and to split the contributions from the new stabilization techniques w.r.t. matrix-vector applications and local assembling routines.

Additionally, we have successfully treated the case of nonlinear viscosity flow models for quasi-Newtonian Power Law approaches. Since in that case the corresponding viscosity parameter $v=v\left(D_{I I}(u)\right)$ depends on the solution $u$ itself, the corresponding stabilization parameter has to be locally adapted since the resulting (local) viscosity $v$ may exhibit significant changes of several orders of magnitude. Nevertheless, the new stabilization techniques lead to very robust and accurate approximation results, and the nonlinear and particularly the linear multigrid solvers show the typical excellent convergence behaviour.

Altogether, we have realized appropriate discretization and efficient solution concepts for nonconforming finite elements which will be able to simulate more complex flow models including the deformation tensor for realistic geometries and configurations. Nevertheless, further research is required and we are currently concentrating on the following aspects:

- Improved locally adaptive stabilization parameters which take into account the quantitative changes due to the nonlinear solution behaviour.

- Better nonlinear Newton-like solvers which are adapted to the additional nonlinearity with regard to the nonlinear stabilization parameters.

- More robust and efficient smoothing operators which use adaptive patching in the case of anisotropic mesh details (see [15]).

\section{REFERENCES}

1. J. Baranger and K. Najib, Analyse numerique des ecoulements quasi-newtoniens dont la viscosity obeit a la loi puissance ou la loi de carreau. Numer. Math. (1990) 58, 35-49.

2. R. Becker, and R. Rannacher, Finite element discretization of the Stokes and Navier-Stokes equations on anisotropic grids. In: Proc. 10th GAMM-Seminar, Kiel, January 14-16, 1994 (G. Wittum, W. Hackbusch, eds.), Volume 49 of Notes on Numerical Fluid Mechanics, Vieweg, 1995, pp. 52-62.

3. S. Brenner, Korn's inequalities for piecewise $H^{1}$ vector fields. IMI preprint series, University of South Carolina, 2002:05 (2002).

4. S.H.M. Buijssen, and S. Turek, Sources of parallel inefficiency for incompressible CFD simulations. In: Proc. 8th International Euro-Par Conference Paderborn, Germany, August 27-30, 2002 (B. Monien, R. Feldmann, eds.), Volume 2400 of Lecture Notes in Computer Science, Springer, 2002.

5. P. Hansbo, and M. G. Larson, A simple nonconforming bilinear element for the elasticity problem. CIMNE, Barcelona, Spain 2001.

6. P. Hansbo and M. G. Larson, Discontinous Galerkin methods for incompressible and nearly incompressible elasticity by Nitsche's method. Preprint 2000-06, Chalmers Finite Element Center.

7. J.Heywood, R. Rannacher and S. Turek, Artificial boundaries and flux and pressure conditions for the incompressible Navier-Stokes equations. Int. J. Numer. Meth. Fluids (1996) 22, 325-352. 
8. J. Hron, J. Malek and S. Turek, A numerical investigation of flows of shear-thinning fluids with applications to blood flow. Int. J. Numer. Meth. Fluids (2000) 32, 863-879.

9. T. J. R. Hughes, The Finite Element Method: Linear Static and Dynamic Finite Element Analysis. Prentice-Hall, New Jersy, 1987.

10. P. Knobloch, On Korn's inequality for nonconforming finite elements. Technische Mechanik (2000) 20, 205-214.

11. P. Knobloch, Discrete Friedrichs' and Korn's inequalities in two and three dimensions. East-West J. Numer. Math. (1996) 4, 35-51.

12. R. Rannacher and S. Turek, A simple nonconforming quadrilateral Stokes element. Numer. Meth. Part. Diff. Equ. (1992) 8, 97-111.

13. D. Schaeffer, Instability in the evolution equations describing incompressible granular flow. $J$. Diff. Eq. (1987) 66, 19-50.

14. M. Schäfer, and S. Turek, Benchmark computations of laminar flow around cylinder. In: Flow Simulation with High-Performance Computers II (E.H. Hirschel editor). Volume 52 of Notes on Numerical Fluid Mechanics, Vieweg, 1996, pp. 547-566.

15. S. Turek, Efficient solvers for incompressible flow problems: An algorithmic and computational approach. LNCSE $\underline{6}$, Springer-Verlag, 1999.

16. S.P. Vanka, Implicit multigrid solutions of Navier-Stokes equations in primitive variables. $J$. Comp. Phys. (1985) 65 138-158. 